\title{
Inclusive DIS Results from HERA
}

\section{Jan Kretzschmar*i}

DESY Zeuthen, Plantanenallee 6, 15738 Zeuthen, Germany

E-mail: jkretz@ifh.de

Deep inelastic $e p$-scattering results and prospects for the two HERA experiments H1 and ZEUS are presented. The topics covered are low and high $Q^{2}$ inclusive structure function measurements, inclusive tagging of heavy quark production in DIS, and QCD fits and parton distribution functions extracted in NLO DGLAP fits. At low $Q^{2}$ improvements and new results are expected due to the better understanding of systematic errors, the low $E_{p}$ run, and combination of ZEUS and H1 data. The high $Q^{2}$ domain profits especially from the increased luminosity and the beam polarisation available after the HERA upgrade. Also for the heavy quark sector improvements are expected due to better statistics and new silicon vertex detectors. A NNLO QCD analysis may lead to better determined parton distribution functions for the LHC and test our understanding of QCD.

DIFFRACTION 2006 - International Workshop on Diffraction in High-Energy Physics

September 5-10 2006

Adamantas, Milos island, Greece

*Speaker.

${ }^{\dagger}$ On behalf of the H1 and ZEUS Collaborations 


\section{Introduction}

The HERA collider facility in Hamburg, Germany, is a unique tool for electron-proton scattering at highest energies. It consists of two accelerators: one for protons, which are accelerated to $920 \mathrm{GeV}$, and one for electrons or positrons ${ }^{1}$, which are accelerated to $27.6 \mathrm{GeV}$. For the two colliding beam experiments H1 and ZEUS this leads to a centre of mass energy of $\sqrt{s}=320 \mathrm{GeV}$.

In Deep Inelastic Scattering (DIS) of leptons off nucleons the substructure of the nucleons was discovered [1]. DIS continues to be a tool for exploring the substructure of the nucleons with the highest precision. The leading order Feynman diagram for neutral current (NC) scattering is given in figure 1 .

The kinematics of the scattering are described in terms of the Lorentz invariant quantities Bjorken scaling variable $x$, inelasticity $y$, and virtuality $Q^{2}$, which are related by $Q^{2}=x y$ s. Figure 1 shows the kinematic $\left(x, Q^{2}\right)$-plane, where the HERA experiments and the fixed target experiments have made measurements of the proton structure. $Q^{2}$ values of up to $50000 \mathrm{GeV}^{2}$ and $x$ values down to $10^{-5}$ are reached at HERA.

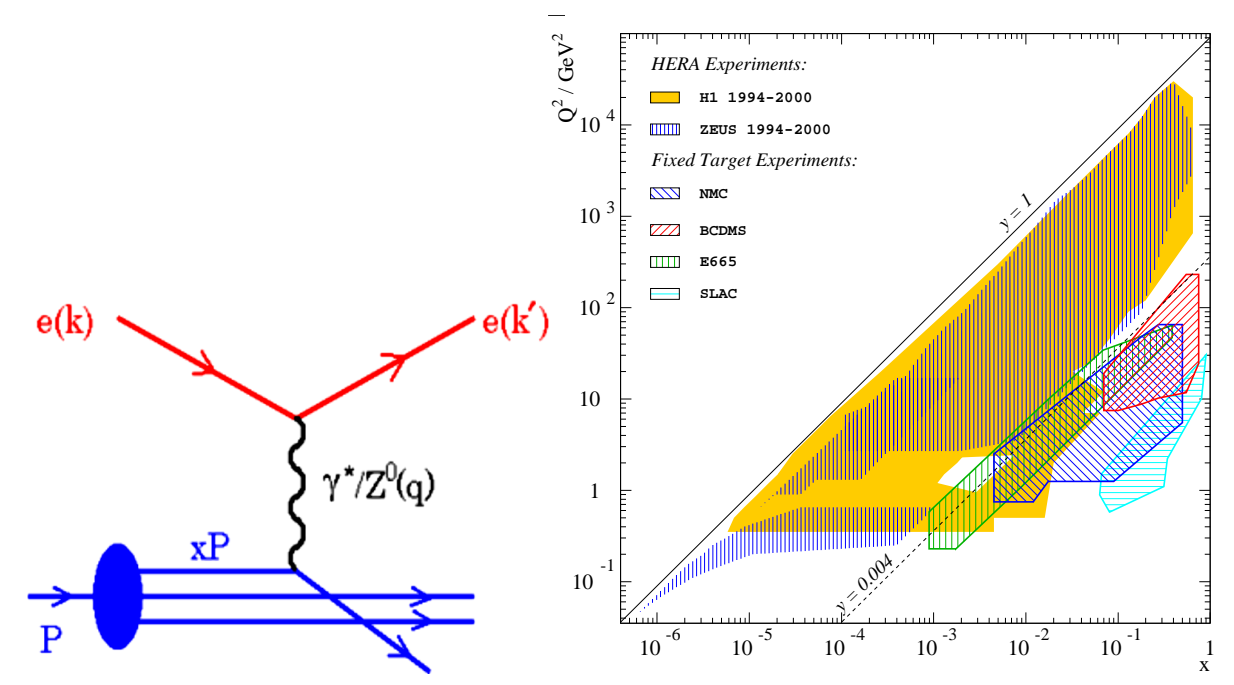

Figure 1: Lowest order Feynman diagram for NC DIS (left). Kinematic plane in $\left(x, Q^{2}\right)$, where measurements of the proton structure function $F_{2}$ have been performed by the HERA collider and fixed target experiments, respectively (right).

One of the most fundamental measurements to be performed is that of the inclusive NC cross section for the reaction $e p \rightarrow e^{\prime} X$, which can be expressed in the form

$$
\frac{\mathrm{d}^{2} \sigma_{N C}^{e^{ \pm} p}}{\mathrm{~d} x \mathrm{~d} Q^{2}}=\frac{2 \pi \alpha^{2} Y_{+}}{x Q^{4}}\left(F_{2}\left(x, Q^{2}\right)-\frac{y^{2}}{Y_{+}} F_{L}\left(x, Q^{2}\right) \mp \frac{Y_{-}}{Y_{+}} x F_{3}\left(x, Q^{2}\right)\right),
$$

with $Y_{ \pm}=1 \pm(1-y)^{2}$ and the structure functions $F_{2}, F_{L}$, and $x F_{3}$.

The structure function $F_{2}$ gives the dominant contribution to the cross section in most of the kinematic domain and therefore has been measured with the best accuracy. Figure 2 gives an example of the progress made with the HERA collider: from the very first data and the discovery of the strong rise of $F_{2}$ towards low $x$ to the currently reached $2-3 \%$ precision in the bulk region.

\footnotetext{
${ }^{1}$ In the following the term electron will usually apply to both lepton charges, if not stated otherwise explicitly.
} 

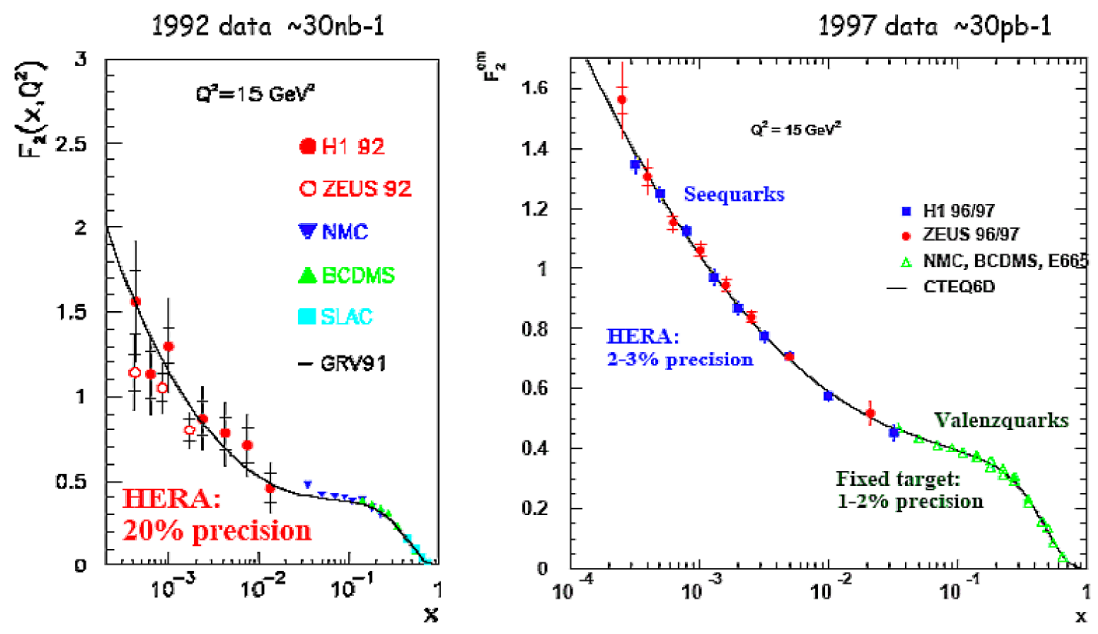

Figure 2: Measurement of the proton structure function $F_{2}$ at $Q^{2}=15 \mathrm{GeV}^{2}$ with the very first HERA data and with much improved precision today [⿰]𠃌 .

The longitudinal structure function $F_{L}$ contributes mostly at high inelasticity $y$, and $x F_{3}$ appears due to neutral weak currents mediated by the $Z$ boson and is only important at high $Q^{2}$.

\section{Results and Prospects for the Low $Q^{2}$ Region}

In the low $Q^{2}$ bulk region $\left(10<Q^{2} / \mathrm{GeV}^{2}<100\right)$ the structure function $F_{2}$ has been measured with an overall precision of $2-3 \%$. In the very low $Q^{2}$ region $\left(Q^{2}<10 \mathrm{GeV}^{2}\right) \mathrm{H} 1$ has obtained preliminary results with special experimental techniques [3, 切:

- runs with shifted vertex to enhance the acceptance of H1 for lower angle and hence lower $Q^{2}$ scattering,

- using events with untagged initial state radiation (ISR) to reach higher $x$ at low $Q^{2}$,

- and runs with a special minimum bias trigger setup.

The preliminary results for the lowest $Q^{2}$ region reached together with measurements from ZEUS and fixed target experiments are shown in figure 3 .

The rise of $F_{2}$ towards low $x$ can be described as $F_{2}\left(x, Q^{2}\right) \propto x^{-\lambda\left(Q^{2}\right)}$. The slope $\lambda\left(Q^{2}\right)$ was extracted from these new results as well as other available data, see figure 3 . At $Q^{2} \lesssim 1 \mathrm{GeV}^{2}$ a change of the slope is observed, as one leaves the DIS regime and enters the domain of nonperturbative QCD effects.

The longitudinal structure function $F_{L}$ contributes to the cross section weighted by the kinematic factor $y^{2} / Y_{+}$. Furthermore it is bound by $0 \leq F_{L} \leq F_{2}$ due to the relations of $F_{2}$ and $F_{L}$ to the absorption cross sections for longitudinal and transversely polarised photons. With data taken at the nominal HERA centre of mass energy, $\mathrm{H} 1$ has performed a model dependant simultaneous extraction of $F_{2}$ and $F_{L}$ from their preliminary low $Q^{2}$ data reaching to high inelasticity $y$ 㺻, see figure $\bigoplus$. The reduced cross section is parameterised as

$$
\sigma_{r}=c\left(Q^{2}\right) \cdot x^{-\lambda\left(Q^{2}\right)}-y^{2} / Y_{+} \cdot F_{L},
$$



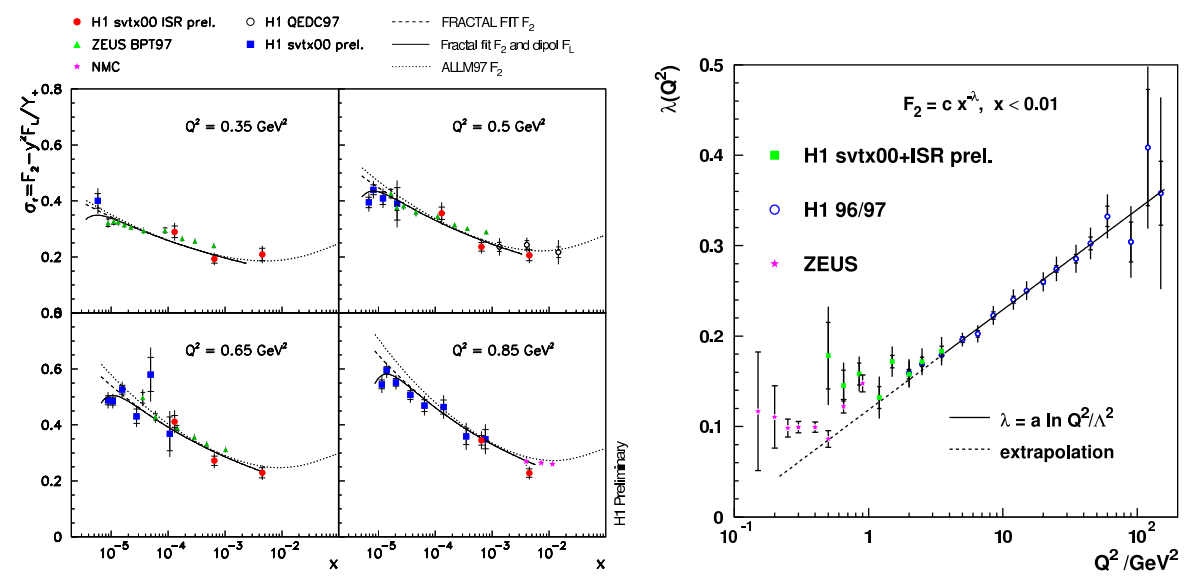

Figure 3: Measurements of the proton structure function $F_{2}$ at $Q^{2}<1 \mathrm{GeV}^{2}$ by ZEUS and recently by H1 from shifted vertex runs and using untagged ISR events (left) and the rise of $F_{2}$ towards low $x$ quantified by $\lambda\left(Q^{2}\right)$ (right) [邚.

and the decrease of $\sigma_{r}$ at low $x$, i.e. high $y$, is attributed to the behaviour of the kinematic factor. One $F_{L}$ value for every $Q^{2}$ bin is thus determined.
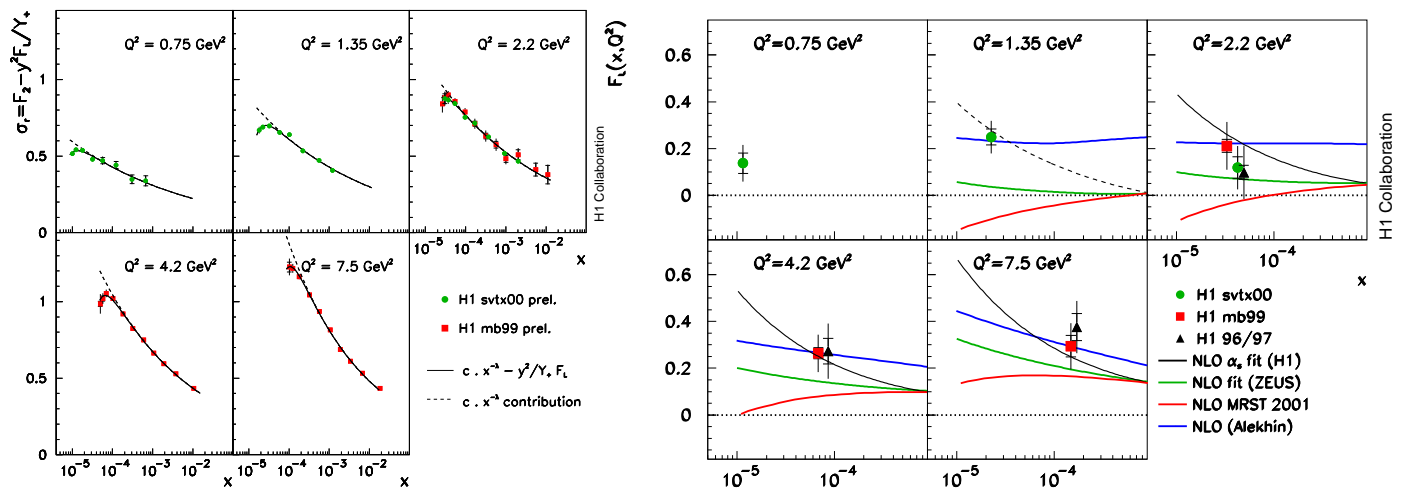

Figure 4: Reduced cross section measurements in the range $1 \lesssim Q^{2} / \mathrm{GeV}^{2}<10$ from special runs (left) and $F_{L}$ determination by the shape method 抑 (right).

To separate the $F_{2}$ and $F_{L}$ contributions to the cross sections without further assumptions, one needs a variation of $y$ at fixed $x$ and $Q^{2}$, which requires a change of the centre of mass energy. For this direct determination of $F_{L}$ a run period of HERA with reduced proton beam energy $E_{p}$ is foreseen in 2007. The simultaneous extraction of the structure functions is illustrated in figure 5 (left). For the accuracy and control of systematics it would be optimal to measure at several centre of mass energies and to cover the range of the kinematic factor $0 \leq f(y)=y^{2} / Y_{+} \leq 1$ as evenly as possible. This also means measuring up to the highest inelasticity $y$ and therefore lowest scattered electron energies. On the other hand each beam energy may require long setup time by the HERA machine group and because the achievable luminosity scales about as $\mathscr{L} \propto E_{p}^{2}$, the amount of different beam energies has to be limited.

One likely scenario at the moment is, that only one additional beam energy of $E_{p}=460 \mathrm{GeV}$, i.e. half of the nominal proton beam energy, will be used and about $\mathscr{L}=10 \mathrm{pb}^{-1}$ will be collected. 

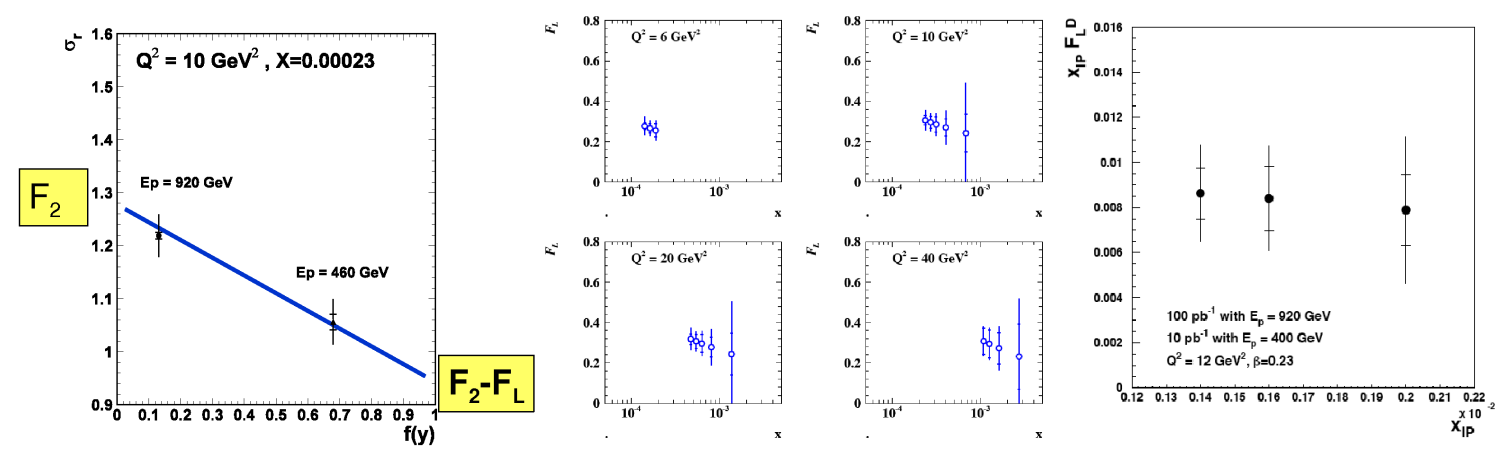

Figure 5: The direct measurement of the structure functions $F_{L}$ and $F_{2}$ from the reduced cross section measurements $\sigma_{r}$ at fixed $x, Q^{2}$ for different inelasticities $y$ (left). Simulated measurement of $F_{L}$ (middle) and $F_{L}^{D}$ (right) with $\mathrm{H} 1$ for the case of one additional low $E_{p}=460 \mathrm{GeV}$ run.

Both experiments, H1 and ZEUS, have studied their capabilities for the measurement. A simulation of the expected measurement of the longitudinal structure function for the inclusive and the diffractive case $\left(F_{L}\right.$ and $F_{L}^{D}$ respectively) by $\mathrm{H} 1$ is given in figure 5. Significant measurement of H1, towards lowest possible $Q^{2}$, and of ZEUS can be expected. Studies to prepare the direct $F_{L}$ measurements are ongoing in H1 and in ZEUS, in close contact with HERA.

$\mathrm{H} 1$ has in the last years invested much work to control the systematics at low $Q^{2}$, which is the limiting factor for precise $F_{2}$ and $F_{L}$ measurements. One advantage of $\mathrm{H} 1$ is the upgraded Backward Silicon Tracker (BST), so precise tracking and charge determination are available not only at higher $Q^{2}$ with the central tracking chamber, but also at lower $Q^{2}$. Using the charge determination of backward going particles, $\gamma p$ background can be separated from the genuine DIS signal at low energies of the scattered lepton. An illustration using data taken in a special run in 1999 is given in figure 6 .

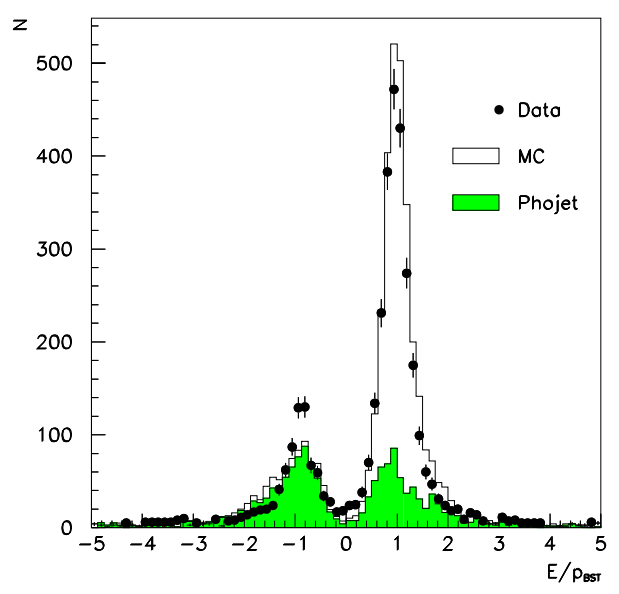

Figure 6: The ratio $E / p$ of the energy measured in the calorimeter $E(4<E / \mathrm{GeV}<10)$ and the signed momentum determined with the $\operatorname{BST} p$. The signal positrons are visible at +1 , while the shaded $\gamma p$ background is about charge symmetric and visible at +1 and -1 .

Detailed calibration and cross checks with $\pi^{0} \rightarrow \gamma \gamma, J / \psi \rightarrow e^{+} e^{-}$, and Compton events $e p \rightarrow$ $e p \gamma$ have limited the residual systematic uncertainty of the lepton energy scale in the backward 
calorimeter of $\mathrm{H} 1$ to $0.2 \%$ at the kinematic peak and $1 \%$ at the lowest energies, see figure 7. Using this knowledge $\mathrm{H} 1$ plans to improve the precision of the $F_{2}$ structure function measurement further in the low $Q^{2}$ bulk and high $y$ region. A further step in precision may be reached by combining ZEUS and $\mathrm{H} 1$ results [阿.
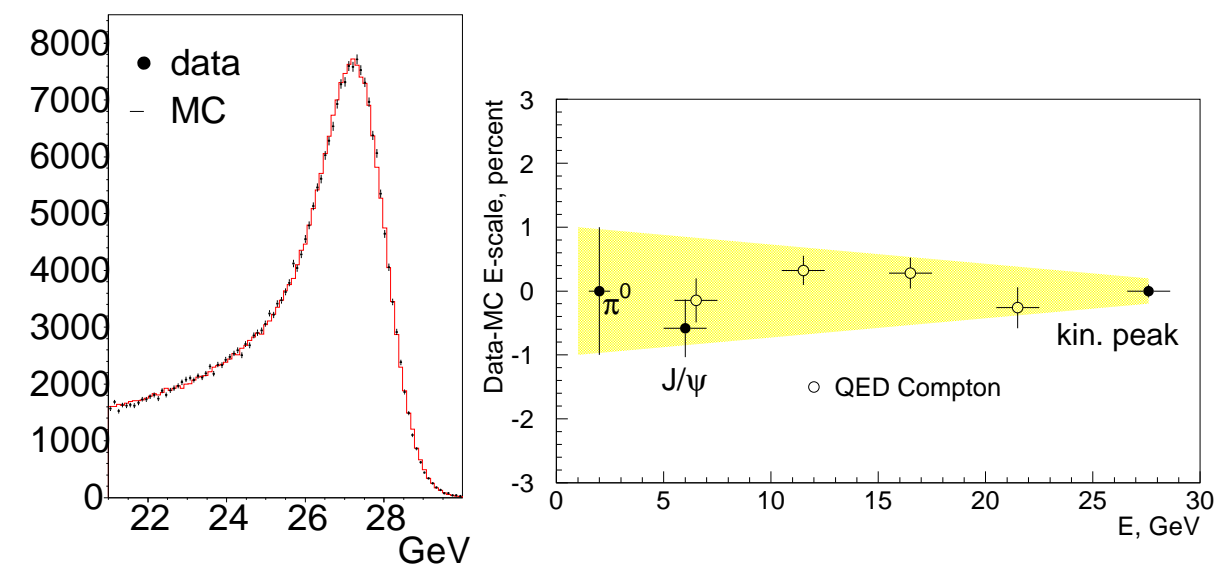

Figure 7: Current preliminary understanding of the lepton energy scale in the backward calorimeter of H1.

\section{Results and Prospects for the High $Q^{2}$ Region}

In figure 8 a collection of $F_{2}$ measurements by $\mathrm{H} 1$ and ZEUS in HERA I, and by fixed target experiments is shown. The errors in the highest $Q^{2}$ domain are still dominated by the statistics. With the data from the current HERA II running phase these errors will be significantly reduced. Also the lepton beams are longitudinally polarised, where a typical polarisation of $35 \%$ is achieved. The spectrum of measurements at high $Q^{2}$ has thus now broadened to charged and neutral currents (CC and NC) with both charge and helicity states of the lepton. Combining these results, tests of the electroweak theory and parton flavour decomposition within one experiment will be possible. First results were obtained already and will be highlighted here.

\subsection{Neutral Currents at High $Q^{2}$}

For polarised beams the structure functions need to be generalised, while the form of the reduced NC inclusive cross section stays the same. The structure function $x \tilde{F}_{3}$ can now no longer be ignored:

$$
\tilde{\sigma}_{N C}^{ \pm}=\frac{\mathrm{d}^{2} \sigma_{N C}^{e^{ \pm} p}}{\mathrm{~d} x \mathrm{~d} Q^{2}} \frac{x Q^{4}}{2 \pi \alpha^{2} Y_{+}}=\tilde{F}_{2}-\frac{y^{2}}{Y_{+}} \tilde{F}_{L} \mp \frac{Y_{-}}{Y_{+}} x \tilde{F}_{3} .
$$

The generalised structure functions receive contributions from $\gamma$ and $Z$ exchange and $\gamma Z$ interference. While at leading order the $\tilde{F}_{2}$ contributions are given by sums of quark and antiquark distributions times the appropriate quark-lepton couplings, $x \tilde{F}_{3}$ is proportional to the difference of quarks and antiquarks and hence sensitive to valence quarks and possible differences of the sea and anti-quark distributions.

The new HERA II data is being analysed by both collaborations. Both single and double differential results have been obtained [6,7]. One example for double differential NC cross sections 

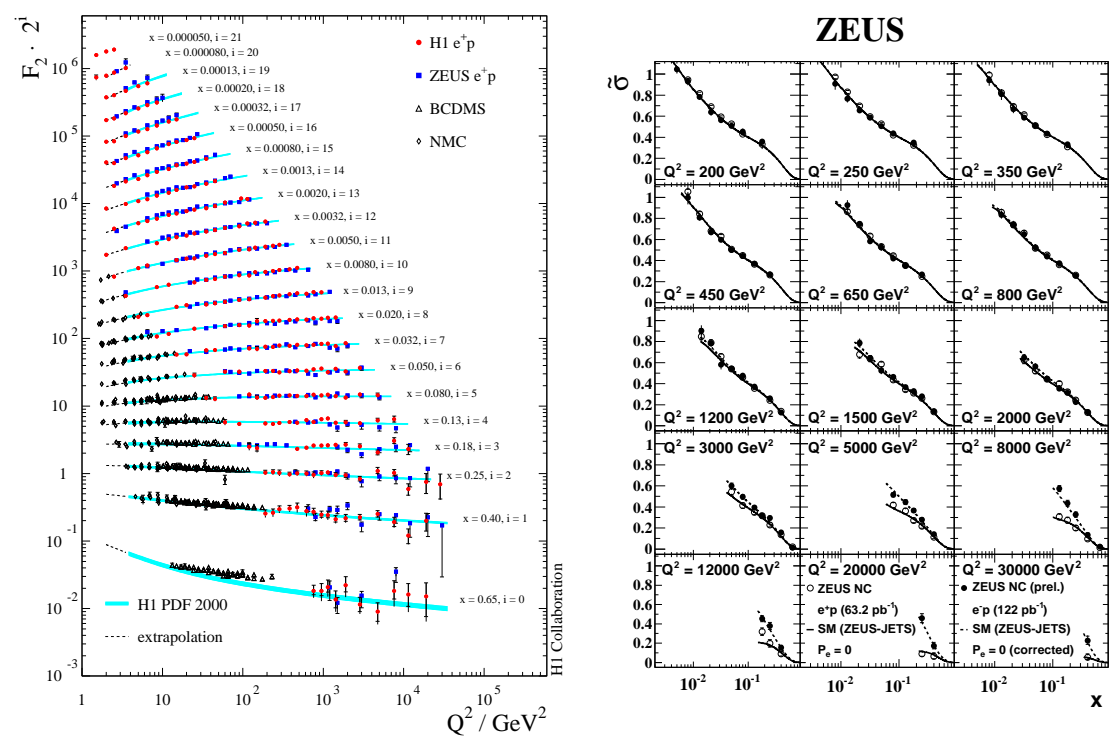

Figure 8: Left: A collection of $F_{2}$ points measured by H1, ZEUS, and fixed target experiments measured so far. Right: New measurements of the unpolarised NC double differential cross sections for $e^{+} p$ and $e^{-} p$ scattering [6].

in $e^{+} p$ and $e^{-} p$ scattering is given in figure 8 (right). These new results have been used to extract the structure function $x \tilde{F}_{3}$ and the polarisation asymmetry $A^{ \pm}$. To increase the statistical significance, $\mathrm{H} 1$ and ZEUS have obtained for the first time combined results using a total luminosity of $480 \mathrm{pb}^{-1}$ [8]. These are summarised in the following.

The structure function $x \tilde{F}_{3}$ is determined from the difference of unpolarised $e^{+} p$ and $e^{-} p \mathrm{NC}$ cross sections:

$$
x \tilde{F}_{3}=-\frac{Y_{+}}{2 Y_{-}}\left(\tilde{\sigma}_{N C}^{+}-\tilde{\sigma}_{N C}^{-}\right)
$$

For measurements performed with polarised beams, data sets with both polarisations are combined and the small residual polarisation is corrected for. The $\gamma Z$ interference is the dominant process, hence further results are given only for $x \tilde{F}_{3}^{\gamma Z}$. Figure 9 shows the results obtained by H1, ZEUS and their combination. A significant reduction of errors can be seen. As $x \tilde{F}_{3}^{\gamma Z}$ is only weakly $Q^{2}$ dependent, all measurements have been combined at $Q^{2}=1500 \mathrm{GeV}^{2}$. The interpretation in terms of quark densities

$$
x F_{3}^{\gamma Z}=\frac{x}{3}\left(2 u_{v}+d_{v}+\Delta\right)
$$

shows the sensitivity to the valence quarks $u_{v}$ and $d_{v}$, and a (small) sea asymmetry $\Delta=2\left(u_{\text {sea }}-\right.$ $\bar{u}+c-\bar{c})+\left(d_{\text {sea }}-\bar{d}+s-\bar{s}\right)$, which conventionally is assumed to vanish.

Similar one can determine a polarisation asymmetry $A^{ \pm}$using the polarised $e_{R L}^{ \pm} p \mathrm{NC}$ cross sections:

$$
A^{ \pm}=\frac{2}{P_{R}-P_{L}} \cdot \frac{\sigma^{ \pm}\left(P_{R}\right)-\sigma^{ \pm}\left(P_{L}\right)}{\sigma^{ \pm}\left(P_{R}\right)+\sigma^{ \pm}\left(P_{L}\right)}
$$

The asymmetries $A^{ \pm}$directly measure the parity violation as they are proportional to a product of 
HERA
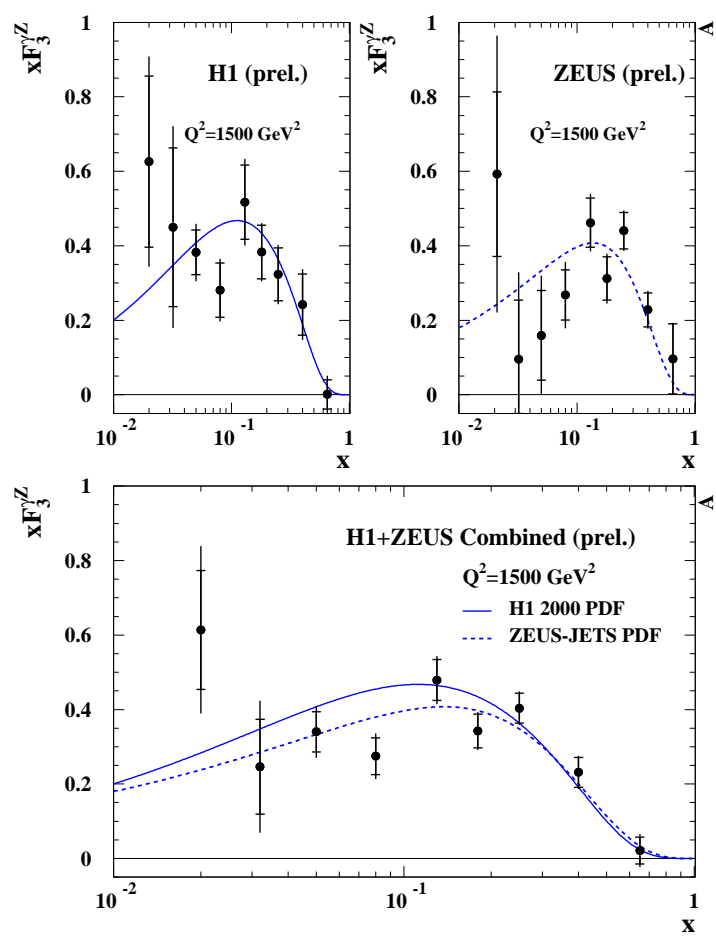

HERA
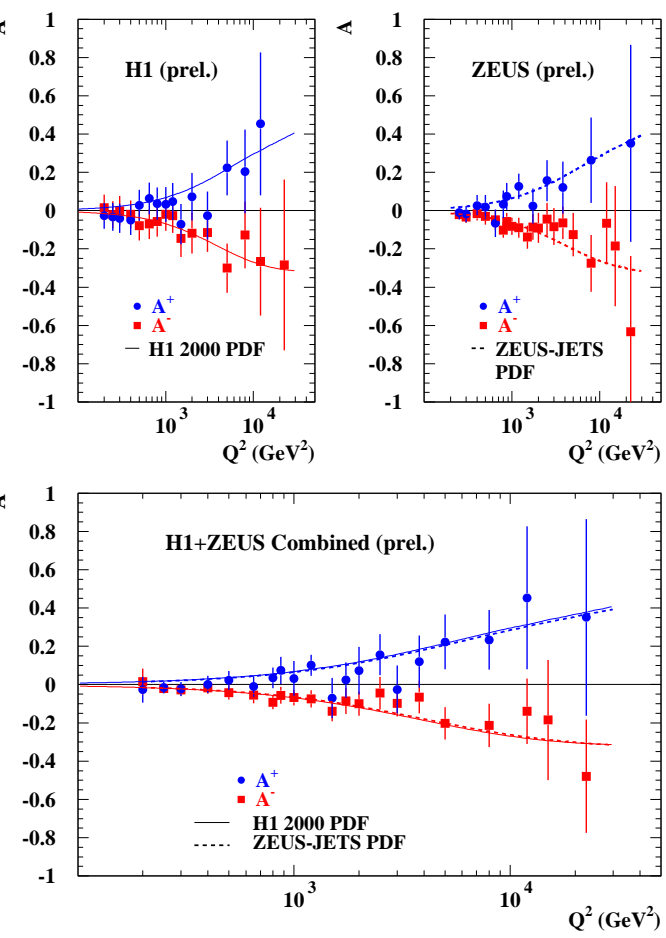

Figure 9: Determination of $x \tilde{F}_{3}^{\gamma Z}$ (left) and the polarisation asymmetries $A^{ \pm}$(right) by H1, ZEUS, and the combination of both [8].

vector and axial vector couplings

$$
A^{ \pm} \simeq k a_{e} \frac{F_{2}^{\gamma Z}}{F_{2}} \propto a_{e} v_{q}
$$

The combined result, see figure 9, is the most significant confirmation of parity violation in DIS to date.

\subsection{Charged Currents at High $Q^{2}$}

The CC cross section is sensitive to the lepton beam charge and depends linearly on the longitudinal lepton polarisation $P_{l}$ :

$$
\frac{\mathrm{d}^{2} \sigma_{C C}^{e^{ \pm} p}}{\mathrm{~d} x \mathrm{~d} Q^{2}}=\left(1 \pm P_{l}\right) \frac{G_{F}^{2}}{2 \pi x}\left(\frac{M_{W}^{2}}{Q^{2}+M_{W}^{2}}\right) \phi_{C C}^{ \pm} .
$$

The functions $\phi_{C C}^{ \pm}$contain the quark density functions and are sensitive to the down-type and up-type quarks in the proton with $e^{+}$and $e^{-}$beams respectively. The total, single, and double differential CC cross sections have been measured using the latest data from HERA II [9, 10, 11]. The total cross section dependence on the lepton beam polarisation shown in figure 10, confirms the chiral structure of the Standard Model, where the $W$ boson only couples to left handed fermions.

In the future improvements for all the measurements at high $Q^{2}$ are expected, once the full HERA I and II data sets are analysed. As of August 2006 the luminosities with $e^{+}$and $e^{-}$beams 


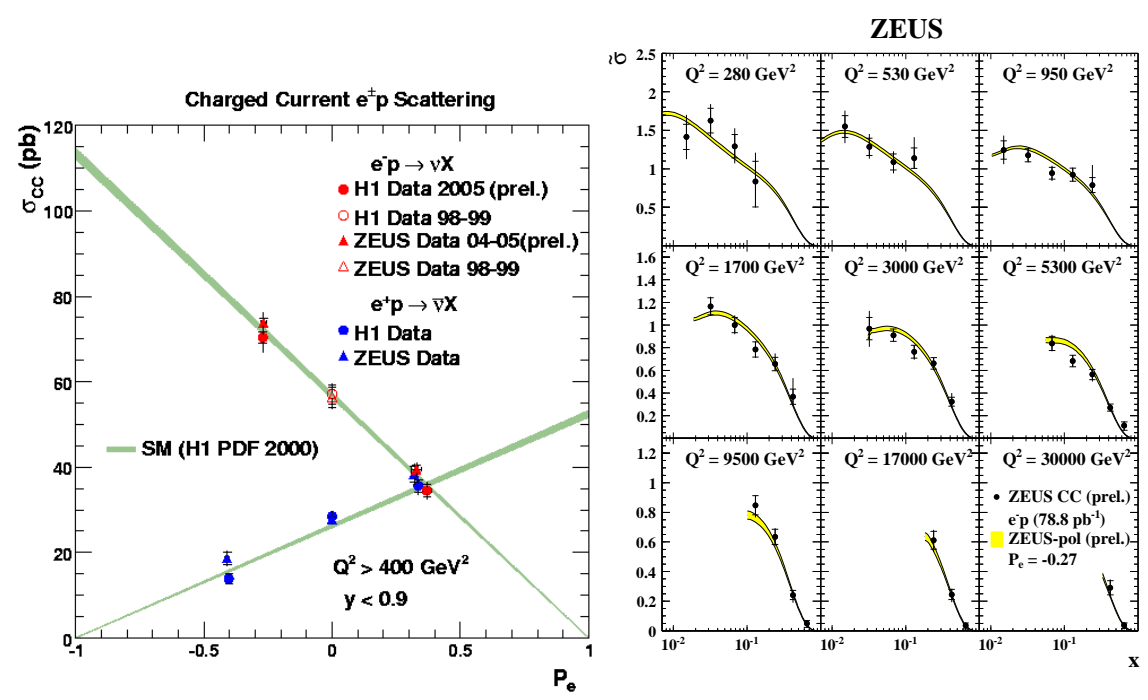

Figure 10: A collection of total $\mathrm{CC}$ cross sections as a function of the beam polarisation as measured by $\mathrm{H} 1$ and ZEUS (left). New polarised double differential CC results by the ZEUS collaboration (right).

are about balanced with $\sim 170 \mathrm{pb}^{-1}$ physics data per beam charge and experiment. At least further $100 \mathrm{pb}^{-1}$ of high energy $e^{+} p$ data are expected before the low energy run will be carried out. The HERA program will then be terminated in the middle of 2007.

\section{Heavy Quark Production in DIS}

The production of heavy flavours in DIS can be calculated using perturbative QCD (pQCD). The problem is well suitable for perturbative calculations as there are multiple hard scales available, i. e. the heavy quark mass $m_{q}$, the transverse momentum of the produced jet $p_{t}$, and of course $Q^{2}$. It is however not clear, which of these scales is most relevant or if some combination should be chosen. First NNLO calculations have become available recently [12]. Various schemes for the mass treatment of the heavy quarks especially in the threshold region are performed and their validity must be checked by comparison to experiment. As the Boson-Gluon fusion process is the dominant heavy quark production process at HERA, the measurement gives a complementary access to the gluon density.

The heavy flavour tagging can be done using exclusive states like $D^{*}$ mesons produced in the charm fragmentation or muons from the semileptonic decays of the heavy quarks. A more inclusive approach is available with high resolution silicon detectors. H1 has recently published results obtained with the Central Silicon Tracker (CST) [13, 14]. For each track in the hadronic final state a signed impact parameter $\delta$ is determined. Large values are rejected as they are likely to be caused by long lived strange particles. Then the significance distributions $S_{i}=\delta_{i} / \Delta \delta_{i}$ of the track with the $i$-th highest significance are evaluated, see figure 11. An enhancement of the heavy quark contributions can be seen at high significance values due to the decay length of the charm and beauty hadrons. The light flavours background distribution on the other hand is smeared due to resolution effects nearly symmetric around zero and can be reduced by subtracting the negative 
from the positive part of the distribution. The fractional contributions of the heavy quarks are then determined by scaling the MC predictions for the light and heavy quark processes to fit the data.
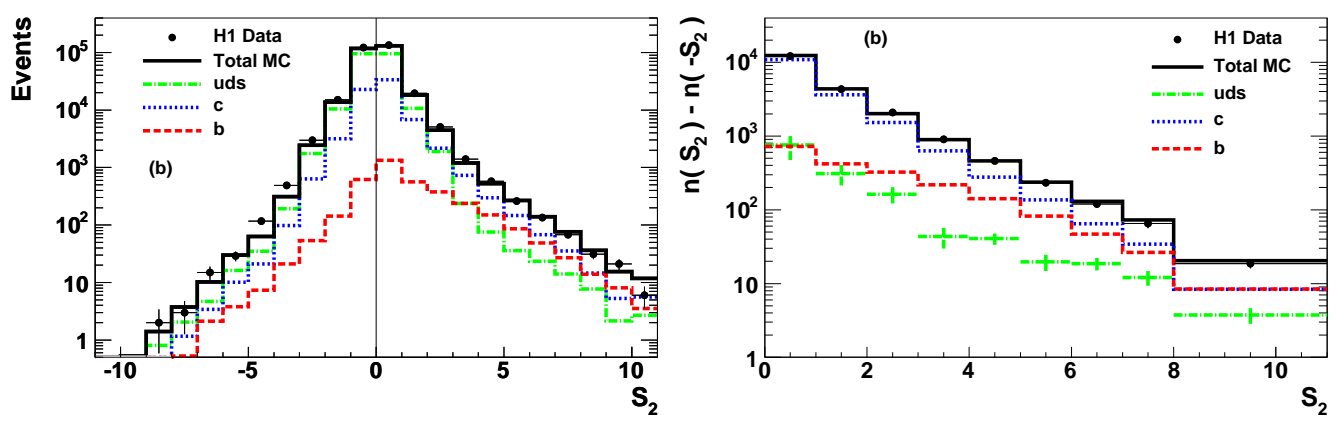

Figure 11: Significance of the track with the second highest significance $S_{2}$ together with the scaled MC distributions for light flavour $(u d s)$, charm $(c)$, and beauty $(b)$ contributions on the left. The right hand side is obtained by subtracting the negative from the positive part of the distribution to reduce the influence of resolution effects.

From the reduced cross sections of the reaction $e p \rightarrow e c \bar{c} X$ the structure functions $F_{2}^{c \bar{c}}$ and $F_{L}^{c \bar{c}}$ are defined analogous to the inclusive case as

$$
\sigma_{r}^{c \bar{c}}=F_{2}^{c \bar{c}}-y^{2} / Y_{+} \cdot F_{L}^{c \bar{c}}
$$

and equivalent for the beauty contribution $(b \bar{b})$. Up to now the published results are based on HERA I data taken by H1. The $F_{2}^{c \bar{c}}$ measurements obtained by this technique and exclusive methods and the first determination of $F_{2}^{b \bar{b}}$ are shown in figure 12.
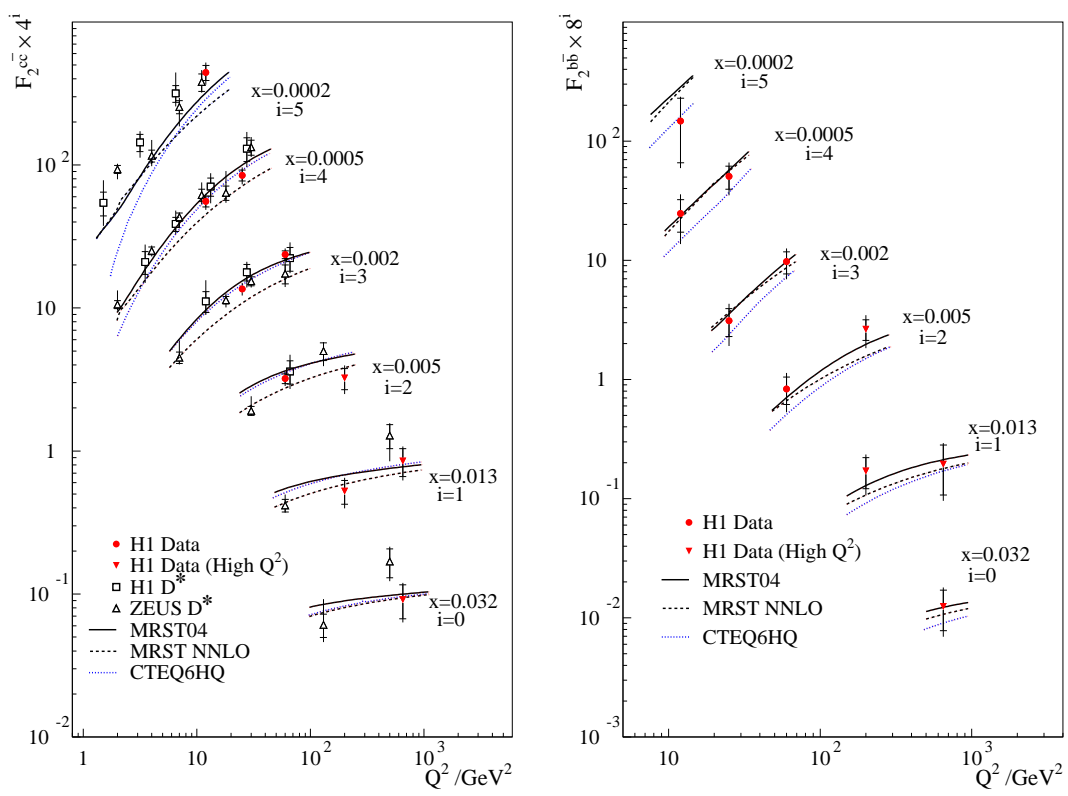

Figure 12: Determination of the heavy quark contributions to DIS with the impact parameter method by H1 and quantified by the structure functions $F_{2}^{c \bar{c}}$ and $F_{2}^{b \bar{b}}[13$, , 14]. 
The charm contribution at low $x$ and $Q^{2}$ is usually of the order $25 \%$ of the total DIS cross section. The beauty contribution is smaller with only about $1 \%$ and depends strongly on $Q^{2}$. The production of heavy flavours is described by $\mathrm{PQCD}$ within the present measurement accuracy. For the HERA II phase ZEUS has installed a "Micro Vertex Detector" and H1 has upgraded their silicon tracking in the forward and backward region. Both collaborations are in the process of understanding the alignment of their new detectors and analysing the new data. An improved precision is expected from the measurements performed using the data collected at HERA II.

\section{QCD Fits and Parton Distribution Functions}

Measuring DIS inclusive cross sections always serves the twofold purpose of determining the parton distribution functions (PDFs), and testing our understanding of the theory of strong interaction, QCD. For these goals it is necessary to analyse the structure functions in the framework of QCD fits. The scaling violations are predicted by the DGLAP equations and the flavours can be separated using fixed target deuteron data. High $Q^{2} \mathrm{CC}$ data from the HERA experiments also probe the flavour content.

Both H1 and ZEUS collaborations have performed this analyses with special emphasis on their own data. The results are shortly reviewed here and differences in the assumptions, input data, and goals are emphasised. All are based on solving NLO DGLAP equations and all give a good description of their input data in terms of $\chi^{2} /$ n.d.f.

\subsection{H1 Gluon and $\alpha_{s}$ fit using inclusive DIS HERA I + BCDMS Data}

The goal of this fit [2] was to determine the gluon distribution $x g\left(x, Q^{2}\right)$ using H1 data (HERA I, $Q^{2}<3000 \mathrm{GeV}^{2}$ ) alone from the scaling violations. A simultaneous determination of flavour separated parton distributions was not aimed at in order to keep the number of fit parameters small. Therefore the fitted distributions were chosen as $x g, V=\frac{9}{4} u_{v}-\frac{3}{2} d_{v}$, and $A=\bar{u}-\frac{1}{4}\left(u_{v}-2 d_{v}\right)$ (valence and sea-like distributions). Together with additional BCDMS data, a competitive determination of the strong coupling constant $\alpha_{s}$ is possible and yields:

$$
\alpha_{s}\left(M_{Z}^{2}\right)=0.1150 \pm 0.0017(\exp )_{-0.0005}^{+0.0009}(\text { model }) \pm 0.005(\text { ren.scale }) .
$$

The extracted gluon distribution and the $\chi^{2}-\chi_{\min }^{2}$ distribution as a function of $\alpha_{s}$ are shown in figure 13 .

\subsection{PDF Determination by H1 using inclusive DIS HERA I Data}

Another fit subsequently performed by H1 is the so called H1PDF 2000 [15], using HERA I inclusive DIS data. The goal is to determine the flavour separated parton distributions of the proton. The fitted distributions were chosen different from the conventional global analyses: $x g, x U, x \bar{U}$, $x D$, and $x \bar{D}$, where e.g. $x U=x(u+c)$ is the sum of all up-type quarks and similar for the other distributions. This choice of parametrisation reflects directly the quark distribution decomposition of the measured NC and CC cross sections, and it weakens the influence of assumptions about the flavour decomposition of the sea. The obtained results together with fits by the CTEQ and MRST collaborations are shown in figure 14. 

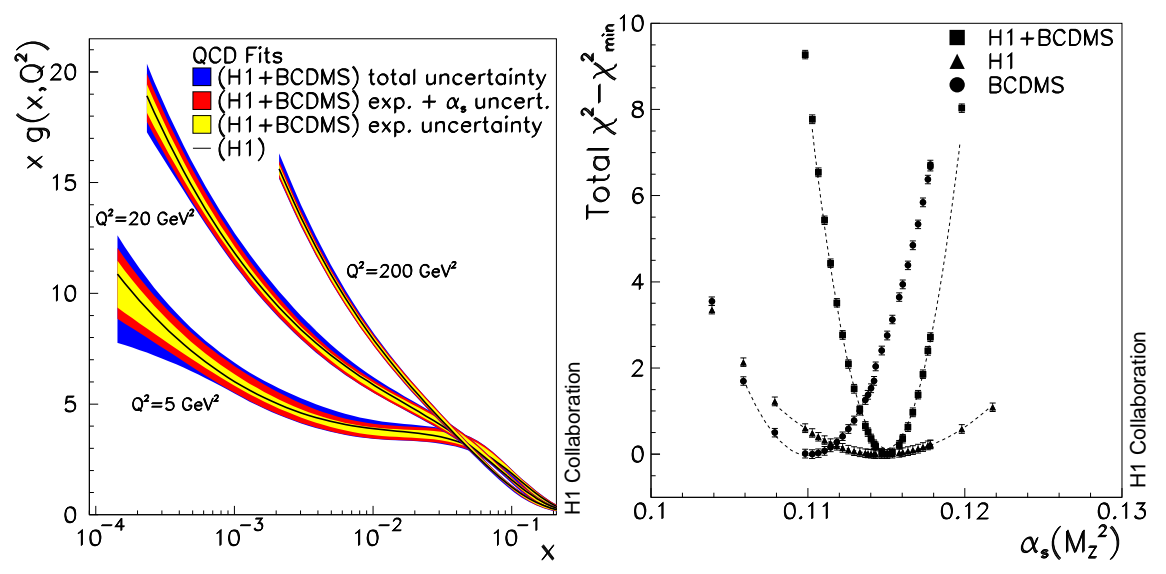

Figure 13: The gluon distribution $x g\left(x, Q^{2}\right)$ and the strong coupling constant $\alpha_{s}$ as determined by $\mathrm{H} 1$ by a NLO QCD fit to DIS data of H1 and BCDMS [月].

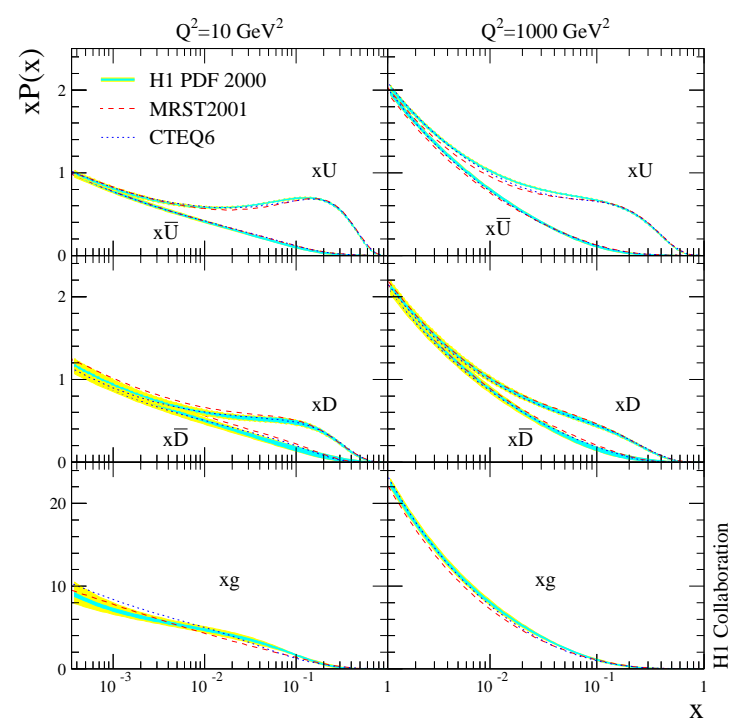

Figure 14: Results of the H1PDF2000 fit [15] in comparison with global fits.

\subsection{ZEUS-S and ZEUS-JETS fits}

The ZEUS collaboration has also published fit results to their DIS data. For the ZEUS-S fit [16] HERA I inclusive DIS data was used together with various fixed target data. In a more recent analysis, the ZEUS-JETS fit [17], jet production data has been added, while the inclusive DIS data from other experiments is not used any more. The fitted distributions in both cases are the more conventional set $x u_{v}, x d_{v}, x S, x g$, and $x(\bar{d}-\bar{u})$. Results of both fits are given in figure 15 .

The addition of the jet data has the advantage, that the gluon evolution can be separated better from the value of the strong coupling constant $\alpha_{s}$. Therefore the fractional uncertainty of the gluon distribution is reduced and a competitive determination of $\alpha_{s}$ is achieved, see figure 16. The result for $\alpha_{s}$ is

$$
\alpha_{s}\left(M_{Z}^{2}\right)=0.1182 \pm 0.0028(\exp ) \pm(0.0008)(\text { model }) \pm 0.005(\text { ren.scale })
$$




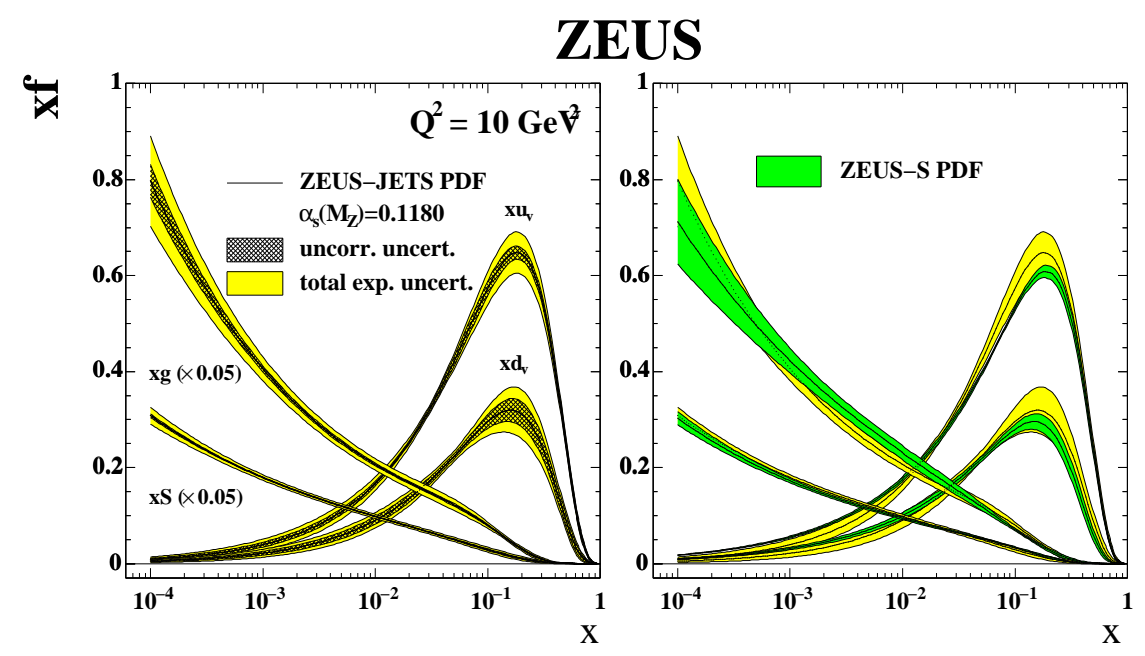

Figure 15: Fit results of the ZEUS-S and ZEUS-JETS analyses [17].
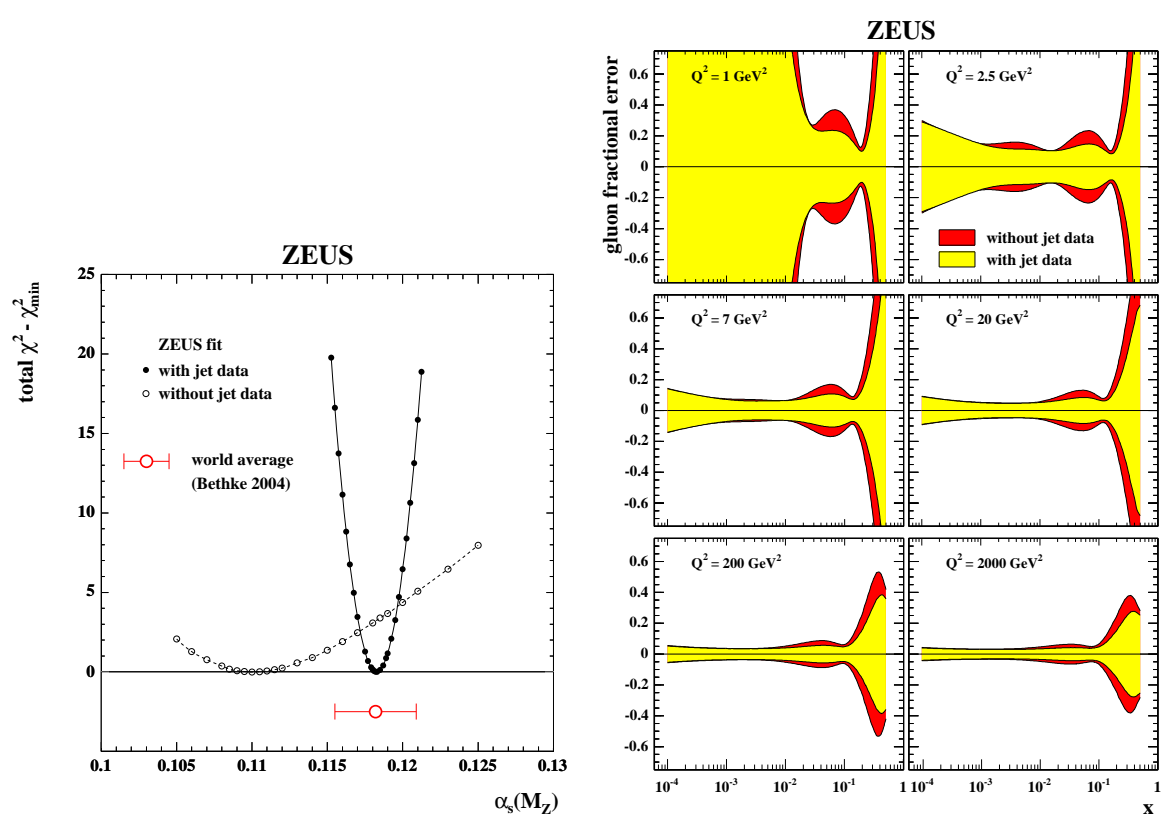

Figure 16: Results of the ZEUS-JETS fit: $\chi^{2}-\chi_{\min }^{2}$ distributions for the $\alpha_{s}$ determination (right) and fractional errors of the gluon distribution for fits with and without the use of jet data [17].

\subsection{ZEUS-pol fit using inclusive DIS + Jet HERA I+II data}

In a preliminary analysis [18], ZEUS has updated the JETS-fit by with polarised high $Q^{2}$ HERA II $e^{-} p$ data to the ZEUS-pol fit. This leads to improved precision for the determination of the valence quark distributions at high $x$, see figure 17. Also fits with two of the electroweak (light) quark couplings $a_{u}, v_{u}, a_{d}, v_{d}$ as free parameters were performed. The results presented in figure 17 are compatible with the Standard Model and are competitive with determinations from other collider experiments. They show an improved precision over the HERA I results published by H1 [19]. 

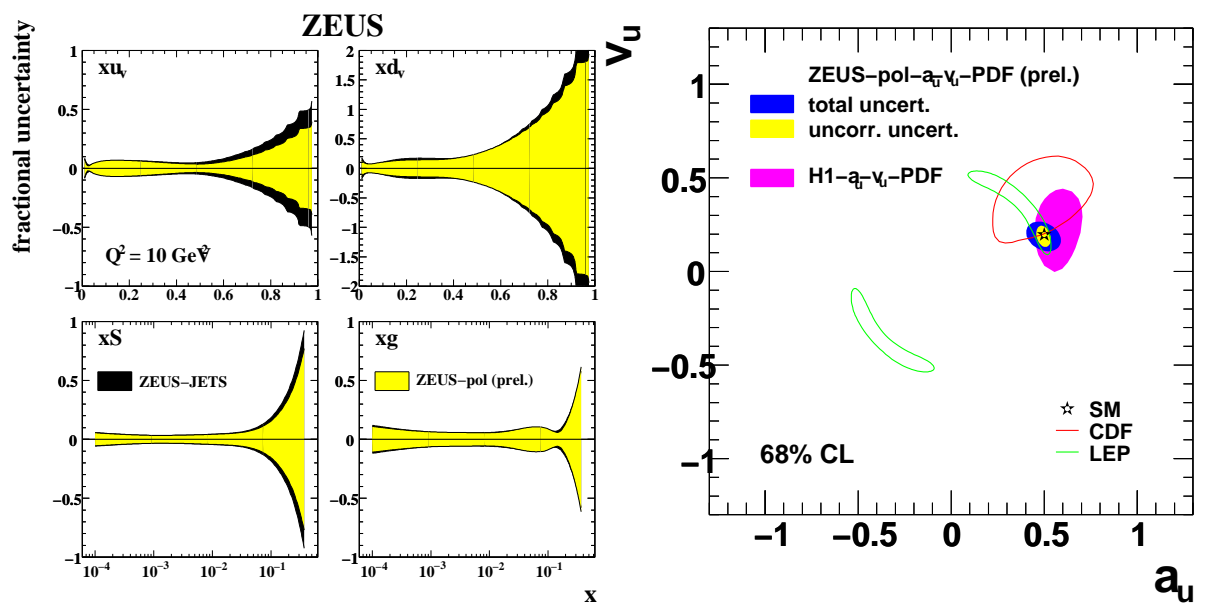

Figure 17: Results of the ZEUS-Pol fits: improved accuracy for the valence quarks at high $x$ (left) and determination of the light quark couplings (right) [18].

\subsection{Status and Prospects for the PDFs}

A precise knowledge of parton distribution functions is essential for predictions and understanding the measurements at the LHC. As can be seen from figure 18, central $(|\eta| \lesssim 2.5)$ production of heavy $(M \sim 100 \mathrm{GeV})$ particles will use all the PDF information obtained in HERA experiments in the range $10^{-4} \lesssim x \lesssim 0.1$. Up to now there has been fair agreement between the PDFs extracted with different approaches. The level of uncertainties can be judged from a comparison of the "standard fits" by H1 and ZEUS, see figure 18. A reasonable agreement as well as some differences can be observed. For further discussion see also [5].
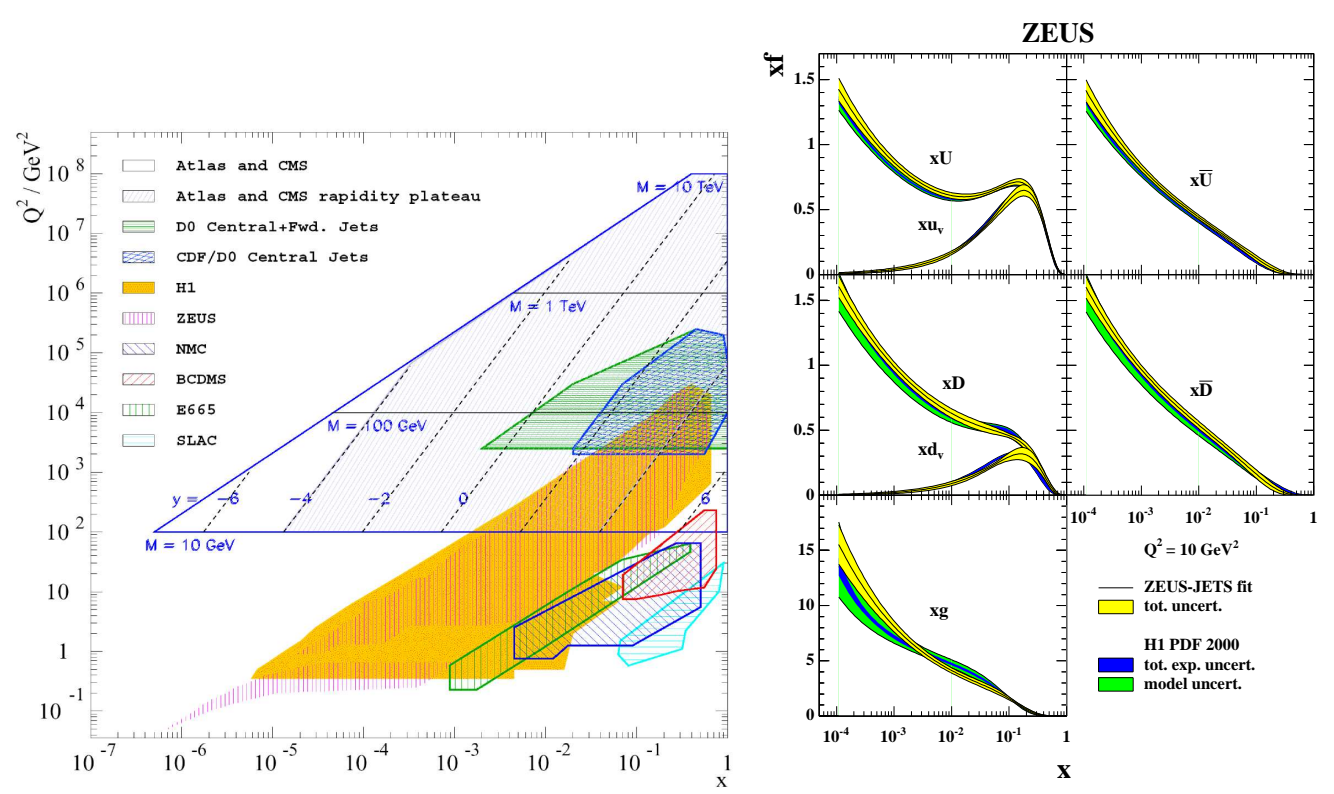

Figure 18: The kinematic plane in $\left(x, Q^{2}\right)$ extended by the measurements of the Tevatron experiments and the region of interest of the LHC. 
To reduce the uncertainties, two things are important. First, further improvements of experimental input is possible in a number of areas. These include the ultimate precision results in the low $Q^{2}$ bulk and high $y$ domain and the direct $F_{L}$ measurement with the run at reduced centre of mass energy. Also the high $Q^{2}$ DIS measurements with high luminosity will enable flavour decomposition within one experiment.

Following the highest experimental precision, further steps have to be taken to fully exploit these measurements. This should include a combination of all HERA data, where a proper treatment of the correlated errors may lead to reduction of systematic uncertainties. A NNLO QCD analysis of the combined data, and cross checks and improvements with semi-inclusive data (e. g. jets and heavy quarks) would be necessary from the theoretical side.

\section{Conclusion and Outlook}

HERA has still potential at low $Q^{2}$ and improved results are expected soon due to a better understanding of the systematic uncertainties. The run at lower proton beam energy $E_{p}$ expected for next year, which will allow to measure the fundamental structure function $F_{L}$ directly for the first time in the HERA domain. With increased luminosity and using the polarised electron and positron beams, new and improved measurements will be possible at high $Q^{2}$. The new silicon detectors will help to determine the heavy quark content of the proton better. Finally a reduction of the PDF uncertainties with improved experimental input, HERA combined data, and NNLO QCD analysis will lead to valuable input for the LHC and will test our understanding of QCD.

\section{References}

[1] M. Breidenbach et al., "Observed Behavior Of Highly Inelastic Electron - Proton Scattering", Phys. Rev. Lett. 23 (1969) 935.

[2] C. Adloff et al. [H1 Collaboration], "Deep-inelastic inclusive e p scattering at low $\mathrm{x}$ and a determination of $\alpha(s)$ ”, Eur. Phys. J. C 21 (2001) 33 [arXiv:hep-ex/0012053].

[3] A. Petrukhin, "New measurement of the structure function $F_{2}\left(x, Q^{2}\right)$ at low $Q^{2}$ with initial state radiation data", H1prelim-04-042, Prepared for 12th International Workshop on Deep Inelastic Scattering (DIS 2004), Strbske Pleso, Slovakia, 14-18 Apr 2004

[4] E. M. Lobodzinska, "Determination of the longitudinal proton structure function $F_{L}$ at low $Q^{2}$ at HERA”, [arXiv:hep-ph/0311180].

[5] S. Alekhin et al., "HERA and the LHC - A workshop on the implications of HERA for LHC physics: Proceedings Part A", 75-113, [arXiv:hep-ph/0601012].

[6] ZEUS Collaboration, "Polarised neutral current DIS cross sections with 2004/05 $e^{-} p$ data and $x F_{3}$ ", ZEUS-prel-06-001, To appear in the proceedings of 33rd International Conference on High Energy Physics (ICHEP 06), Moscow, Russia, 26 Jul - 2 Aug 2006.

[7] H1 Collaboration, "Neutral Current Interactions in $e^{ \pm} p$ Scattering with Longitudinally Polarised Leptons", H1prelim-06-042, To appear in the proceedings of 33rd International Conference on High Energy Physics (ICHEP 06), Moscow, Russia, 26 Jul - 2 Aug 2006. 
[8] H1 and ZEUS Collaborations, "Electroweak Neutral Currents at HERA", H1prelim-06-142, ZEUS-prel-06-022, To appear in the proceedings of 33rd International Conference on High Energy Physics (ICHEP 06), Moscow, Russia, 26 Jul - 2 Aug 2006.

[9] ZEUS Collaboration, "Polarised charged current DIS cross sections with 2004/05 e-p data", ZEUS-prel-06-002, To appear in the proceedings of 33rd International Conference on High Energy Physics (ICHEP 06), Moscow, Russia, 26 Jul - 2 Aug 2006.

[10] A. Aktas et al. [H1 Collaboration], "First measurement of charged current cross sections at HERA with longitudinally polarised positrons”, Phys. Lett. B 634 (2006) 173 [arXiv:hep-ex/0512060].

[11] H1 Collaboration, “ High $Q^{2}$ Charged Current in polarised ep collisions”, H1prelim-06-041, To appear in the proceedings of 14th International Workshop on Deep Inelastic Scattering (DIS 2006), Tsukuba, Japan, 20-24 Apr 2006.

[12] R. S. Thorne, AIP Conf. Proc. 792 (2005) 847 [arXiv:hep-ph/0506251].

[13] A. Aktas et al. [H1 Collaboration], "Measurement of $F_{2}^{c \bar{c}}$ and $F_{2}^{b \bar{b}}$ at high $Q^{2}$ using the H1 vertex detector at HERA", Eur. Phys. J. C 40 (2005) 349 [arXiv:hep-ex/0411046].

[14] A. Aktas et al. [H1 Collaboration], "Measurement of $F_{2}^{c \bar{c}}$ and $F_{2}^{b \bar{b}}$ at low $Q^{2}$ and $x$ using the H1 vertex detector at HERA”, Eur. Phys. J. C 45 (2006) 23 [arXiv:hep-ex/0507081].

[15] C. Adloff et al. [H1 Collaboration], "Measurement and QCD analysis of neutral and charged current cross sections at HERA”, Eur. Phys. J. C 30 (2003) 1 [arXiv:hep-ex/0304003].

[16] S. Chekanov et al. [ZEUS Collaboration], "A ZEUS next-to-leading-order QCD analysis of data on deep inelastic scattering”, Phys. Rev. D 67 (2003) 012007 [arXiv:hep-ex/0208023].

[17] S. Chekanov et al. [ZEUS Collaboration], "An NLO QCD analysis of inclusive cross-section and jet-production data from the ZEUS experiment”, Eur. Phys. J. C 42 (2005) 1 [arXiv:hep-ph/0503274].

[18] ZEUS Collaboration, "QCD and Electroweak analysis of the ZEUS NC and CC inclusive and jet cross sections”, ZEUS-prel-06-003, To appear in the proceedings of 14th International Workshop on Deep Inelastic Scattering (DIS 2006), Tsukuba, Japan, 20-24 Apr 2006.

[19] A. Aktas et al. [H1 Collaboration], "A determination of electroweak parameters at HERA", Phys. Lett. B 632 (2006) 35 [arXiv:hep-ex/0507080]. 